\title{
A functional calculus description of real interpolation spaces for sectorial operators
}

\author{
by
}

Markus HaAse (Pisa)

\begin{abstract}
For a holomorphic function $\psi$ defined on a sector we give a condition implying the identity

$$
\left(X, \mathcal{D}\left(A^{\alpha}\right)\right)_{\theta, p}=\left\{x \in X \mid t^{-\theta \operatorname{Re} \alpha} \psi(t A) \in \mathbf{L}_{*}^{p}((0, \infty) ; X)\right\}
$$

where $A$ is a sectorial operator on a Banach space $X$. This yields all common descriptions of the real interpolation spaces for sectorial operators and allows easy proofs of the moment inequalities and reiteration results for fractional powers.
\end{abstract}

1. Introduction. Sectorial operators (also called "nonnegative operators" sometimes) on Banach spaces have been the object of study for at least the last fifty years. The reason for this interest is that they form a natural abstraction of elliptic differential operators. In addition also generators of (not necessarily holomorphic) semigroups can be subsumed under this concept. In the late fifties, the concept of fractional power $A^{\alpha}$ of a sectorial operator $A$ was invented to replace the discrete scale of regularity coming along with $A$ by a continuous analogue (see [17, Sec. 3.4] for more information). Around the same time, a general theory of so-called real interpolation spaces was created (see [4, Sec. 3.14]). Unfortunately, even in "standard" examples (like the Laplacian on $\mathbf{L}^{p}\left(\mathbb{R}^{d}\right)$ for $p \neq 2$ ) the domains of fractional powers do not coincide with real interpolation spaces $\left(X, \mathcal{D}\left(A^{m}\right)\right)_{\theta, p}$. Moreover, it became clear that real interpolation spaces are much better suited for dealing with regularity questions. (See [15], where the regularity theory for the inhomogeneous Cauchy problem $u^{\prime}+A u=f$ is treated.) One reason for this is that the real interpolation spaces $\left(X, \mathcal{D}\left(A^{m}\right)\right)_{\theta, p}$ allow particularly

2000 Mathematics Subject Classification: 46B70, 47A60, 47D06, 47B99.

Key words and phrases: sectorial operator, functional calculus, real interpolation spaces, corona theorem, Bézout equation.

The author gratefully acknowledges the financial support by the EU-Research Training Network "Evolution Equations for Deterministic and Stochastic Systems", Contract No. HPRN-CT-2002-00281. 
nice descriptions. For $m=1$ one has for example

$$
(X, \mathcal{D}(A))_{\theta, p}=\left\{x \in X \mid\left\|t^{\theta} A(t+A)^{-1} x\right\|_{X} \in \mathbf{L}^{p}((0, \infty) ; d t / t)\right\},
$$

and, if $-A$ generates a bounded holomorphic semigroup,

$$
(X, \mathcal{D}(A))_{\theta, p}=\left\{x \in X \mid\left\|t^{(1-\theta)} A e^{-t A} x\right\|_{X} \in \mathbf{L}^{p}((0, \infty) ; d t / t)\right\}
$$

(see [15, Chapter 2]). In a series of papers, Komatsu explored the fractional powers and in particular studied the relation of the domains $\mathcal{D}\left(A^{\alpha}\right)$ and the real interpolation spaces. He generalized (1) to

$$
\left(X, \mathcal{D}\left(A^{\alpha}\right)\right)_{\theta, p}=\left\{x \in X \mid\left\|t^{\theta \operatorname{Re} \alpha}\left[A(t+A)^{-1}\right]^{\alpha} x\right\|_{X} \in \mathbf{L}^{p}((0, \infty) ; d t / t)\right\},
$$

a description which today bears his name (see [12] or [17, Sec. 11.3]).

A more recent concept developed to study sectorial operators is the functional calculus. Initiated by McIntosh [18] on Hilbert spaces, its generalization to Banach spaces has proved to be a particularly useful tool in understanding phenomena around sectorial operators (see [2] or [14] and the references therein). Using the language of functional calculus, one can see that the above descriptions of real interpolation spaces follow a common pattern, namely

$$
\left(X, \mathcal{D}\left(A^{\alpha}\right)\right)_{\theta, p}=\left\{x \mid\left\|t^{-\theta \operatorname{Re} \alpha} \psi(t A) x\right\|_{X} \in \mathbf{L}^{p}((0, \infty) ; d t / t)\right\}
$$

(see Section 7 for details). Such a description (in case $X$ is a Hilbert space and $p=2$ ) played a decisive role already in McIntosh's work (cp. also [3]), especially in the proof of his fundamental result on the boundedness of the $H^{\infty}$-calculus. (The key property here is that one has a certain freedom in choosing the function $\psi$.) One can generalize the results from [3] in order to find a large class of admissible functions $\psi$. However, this generalization works only in the case when $A$ is injective, whereas the above descriptions (1)-(3) do not require injectivity of $A$. Hence, up to now a general criterion for functions $\psi$ satisfying (4) is missing. In this paper we give such a criterion which is particularly simple in the case $\theta \in(0,1)$ (Theorem 1 ). Imposing stronger conditions also includes the extremal case $\theta=1, p=\infty$ (Theorem 2). Our results contain all the known descriptions and even yield new ones.

The plan of the paper is as follows. In Section 2 we recall some basic definitions and facts about sectorial operators. Also, we sketch the construction of the functional calculus and list those of its properties which will be used in the later sections. In Section 3 we state our main results, Theorems 1 and 2. In Theorem 1 a simple condition on $\psi$ suffices, but one has the identity (4) only for $\theta \neq 1$. In Theorem 2 the extremal case $\theta=1, p=\infty$ is included, but the conditions on the function $\psi$ are more restrictive.

In Section 4 we give a proof of a special case (Theorem 3 ) of Theorem 2 since this proof is easier and particularly interesting. The proof of Theorem 2 
is then given in Section 5. Finally, in Section 6 we present the proof of Theorem 1, which uses many of the ideas developed so far.

In Section 7 we first show that the common formulations (1)-(3) are instances of our generic description (Sections 7.1 and 7.2). Then we show how the well known moment inequalities for fractional powers are derived with our methods, and how one can easily obtain results like

$$
\left(X, \mathcal{D}\left(A^{\alpha}\right)\right)_{\theta \frac{\operatorname{Re} \beta}{\operatorname{Re} \alpha}, p}=\left(X, \mathcal{D}\left(A^{\beta}\right)\right)_{\theta, p}
$$

without using abstract reiteration arguments (Section 7.4). Finally, in Section 7.5 we relate one of our auxiliary results (Theorem 4) to the famous Carleson Corona Theorem.

Here are some definitions and notational conventions. For any open set $\Omega \subset \mathbb{C}$ we denote by $\mathcal{O}(\Omega)$ (resp. $H^{\infty}(\Omega)$ ) the space of all holomorphic (resp. bounded holomorphic) functions on $\Omega$. The function $z \mapsto z$ is abbreviated simply by $z$. For $f \in H^{\infty}(\Omega)$ we define

$$
\|f\|_{\Omega}:=\|f\|_{\infty, \Omega}=\sup \{|f(z)| \mid z \in \Omega\} .
$$

On $\Omega=\mathbb{C} \backslash(-\infty, 0]$ we consider the functions $z^{\alpha}:=e^{\alpha \log z}$ where $\log z$ denotes the principal part of the logarithm.

For an operator $A$ on a Banach space $X$, we denote by $\mathcal{N}(A)$ its kernel and by $\mathcal{R}(A)$ its range. We denote by $\mathcal{L}(X)$ the set of all bounded, everywhere defined operators on $X$. The resolvent set $\varrho(A)$ of $A$ is defined as $\varrho(A):=$ $\left\{\lambda \in \mathbb{C} \mid \lambda-A\right.$ is injective and $\left.(\lambda-A)^{-1} \in \mathcal{L}(X)\right\}$, and we write $R(\lambda, A):=$ $(\lambda-A)^{-1}$ for the resolvent of $A$. The set $\sigma(A):=\mathbb{C} \backslash \varrho(A)$ is the spectrum of $A$. One may consult [7] for other notations and the basic results of spectral theory for unbounded operators. If $A$ and $B$ are any operators on $X$ we define their product $B A$ as $(B A) x:=B(A x)$ for $x \in \mathcal{D}(B A):=\{x \in \mathcal{D}(A) \mid A x \in$ $\mathcal{D}(B)\}$.

Let $(a, b) \subset(0, \infty)$ and $1 \leq p \leq \infty$. We denote by $\mathbf{L}_{*}^{p}((a, b) ; X)$ the Bochner space of (equivalence classes of) $X$-valued functions wich are in $\mathbf{L}^{p}$ with respect to the measure $d t / t$. If $X=\mathbb{C}$, we simply write $\mathbf{L}_{*}^{p}(a, b)$. The positive real coordinate $(t \mapsto t)$ is abbreviated simply by $t$.

2. Sectorial operators and their functional calculus. In this section we briefly recall the definition of sectorial operators and their holomorphic functional calculus.

For $0 \leq \omega \leq \pi$ let

$$
S_{\omega}:= \begin{cases}\{z \in \mathbb{C} \mid z \neq 0 \text { and }|\arg z|<\omega\}, & 0<\omega \leq \pi \\ (0, \infty), & \omega=0\end{cases}
$$

Hence if $\omega>0, S_{\omega}$ denotes the open sector symmetric with respect to the positive real axis with opening angle $\omega$. 
Let $X$ be a Banach space and $\omega \in[0, \pi)$. A (possibly unbounded) operator $A$ is called sectorial of angle $\omega$ (for short: $A \in \operatorname{Sect}(\omega)$ ) if

1) $\sigma(A) \subset \bar{S}_{\omega}$

2) $M\left(A, \omega^{\prime}\right):=\sup \left\{\|\lambda R(\lambda, A)\| \mid \lambda \notin \bar{S}_{\omega^{\prime}}\right\}<\infty$ for all $\omega^{\prime} \in(\omega, \pi)$.

If $A$ is sectorial, we call

$$
\omega_{A}:=\min \{0 \leq \omega<\pi \mid A \in \operatorname{Sect}(\omega)\}
$$

the spectral angle (or sectoriality angle) of $A$. Note that if $A \in \operatorname{Sect}(\omega)$ then also $t A \in \operatorname{Sect}(\omega)$ for each $t>0$, with the same constants: $M\left(t A, \omega^{\prime}\right)=$ $M\left(A, \omega^{\prime}\right)$ for all $\omega<\omega^{\prime} \leq \pi$. More on basic properties of sectorial operators can be found in [17] or [10]. Note that we do not assume a sectorial operator to be densely defined or have dense range, although these density assumptions appear frequently in the literature as part of the definition.

For $\varphi \in(0, \pi]$ we let

$$
H_{0}^{\infty}\left(S_{\varphi}\right):=\left\{f \in \mathcal{O}\left(S_{\varphi}\right)|\exists C, s>0:| f(z) \mid \leq C \min \left(|z|^{s},|z|^{-s}\right)\right\} .
$$

We enlarge this algebra (!) to obtain

$$
\mathcal{E}\left(S_{\varphi}\right):=H_{0}^{\infty}\left(S_{\varphi}\right) \oplus \mathbb{C} \frac{1}{1+z} \oplus \mathbb{C} \mathbf{1} .
$$

A function $f \in \mathcal{O}\left(S_{\varphi}\right)$ is in $\mathcal{E}\left(S_{\varphi}\right)$ if and only if it is bounded, has finite limits at $\{0, \infty\}$ and these limits are approached "polynomially fast". Since

$$
\frac{1}{1+1 / z}=\frac{z}{1+z}=1-\frac{1}{1+z},
$$

one has $f \in \mathcal{E}\left(S_{\varphi}\right)$ if and only if $f\left(z^{-1}\right) \in \mathcal{E}\left(S_{\varphi}\right)$. If the precise sector is understood or not important, we sometimes write just $\mathcal{E}, H_{0}^{\infty}$ instead of $\mathcal{E}\left(S_{\varphi}\right), H_{0}^{\infty}\left(S_{\varphi}\right)$. We will use the following simple fact.

Lemma 2.1. Let $\operatorname{Re} \alpha>0$. Then

$$
\frac{z^{\alpha}}{(1+z)^{\alpha}}, \frac{1}{(1+z)^{\alpha}} \in \mathcal{E}\left(S_{\varphi}\right)
$$

for each $0<\varphi<\pi$.

Proof. One has $z^{\alpha} w^{\alpha}=(z w)^{\alpha}$ whenever $z, w, z w \in S_{\pi}$. Therefore

$$
\frac{1}{\left(1+z^{-1}\right)^{\alpha}}=\left(\frac{1}{1+z^{-1}}\right)^{\alpha}=\left(\frac{z}{1+z}\right)^{\alpha}=\frac{z^{\alpha}}{(1+z)^{\alpha}}
$$

for all $z \in S_{\pi}$. Hence it suffices to show that $1 /(1+z)^{\alpha} \in \mathcal{E}\left(S_{\varphi}\right)$. To see this, note first that this function decays polynomially fast at $\infty$. Second, since the function $z^{\alpha}$ is holomorphic at 1 , the function $1 /(1+z)^{\alpha}$ is holomorphic at 0 . Hence there is $C>0$ such that

$$
\left|\frac{1}{(1+z)^{\alpha}}-1\right| \leq C|z| \quad(z \text { near } 0),
$$

which completes the proof. 
Given a function $f(z):=\psi(z)+\frac{c}{1+z}+d \mathbf{1} \in \mathcal{E}\left(S_{\varphi}\right)$ and a sectorial operator $A$ with $\omega_{A}<\varphi$ one defines

$$
\Phi(A):=f(A):=\psi(A)+c(1+A)^{-1}+d \in \mathcal{L}(X),
$$

where $\psi(A)$ is defined by a Cauchy integral

$$
\psi(A):=\frac{1}{2 \pi i} \int_{\partial S_{\omega^{\prime}}} \psi(z) R(z, A) d z
$$

for (any) $\omega^{\prime} \in(\omega, \varphi)$. This establishes an algebra homomorphism $\Phi: \mathcal{E}\left(S_{\varphi}\right)$ $\rightarrow \mathcal{L}(X)$. Details of this construction can be found in [9] and [10]. Only a few properties of the functional calculus will be used in this paper. We list these properties in the rest of this section.

Lemma 2.2. Let $A \in \operatorname{Sect}(\omega), \varphi \in(\omega, \pi)$ and $f \in \mathcal{E}\left(S_{\varphi}\right)$. Then the function $(t \mapsto f(t A)):(0, \infty) \rightarrow \mathcal{L}(X)$ is continuous and

$$
C_{f}:=\sup _{t>0}\|f(t A)\|<\infty .
$$

Proof. Write $f=\psi+c(1+z)^{-1}+d$. The uniform boundedness of the family $\left((1+t A)^{-1}\right)_{t>0}$ is just the definition of sectoriality. The uniform boundedness of $(\psi(t A))_{t>0}$ follows from a simple change of variables in the Cauchy integral. Continuity is also straightforward.

There is a standard way to extend the "elementary" functional calculus $\Phi: \mathcal{E}\left(S_{\varphi}\right) \rightarrow \mathcal{L}(X)$ to a larger algebra of functions on the sector $S_{\varphi}$ (see [5], [1] or [10]). The operators so obtained may then be unbounded. In this way one can define the fractional powers $A^{\alpha}$ for all $\operatorname{Re} \alpha>0$. A general fact in the extended functional calculus is the identity $(f g)(A)=f(A) g(A)$ whenever $g(A) \in \mathcal{L}(X)$ (see one of the above references or [9]). As a special case of this we note the following.

Lemma 2.3. Let $\operatorname{Re} \alpha>0$ and $f, z^{\alpha} f \in \mathcal{E}\left(S_{\varphi}\right)$. Then $\mathcal{R}(f(A)) \subset \mathcal{D}\left(A^{\alpha}\right)$ and $A^{\alpha} f(A)=\left(z^{\alpha} f\right)(A)$.

Another basic fact is that $f(t A)=[f(t z)](A)$ for all $f$ from a reasonably large class of functions, containing in particular all functions $f \in \mathcal{E}$ and all the fractional powers $f=z^{\alpha}$ with $\operatorname{Re} \alpha>0$. This readily yields $(t A)^{\alpha}=$ $t^{\alpha} A^{\alpha}$ and $\left.\mathcal{D}\left((t A)^{\alpha}\right)\right)=\mathcal{D}\left(A^{\alpha}\right)$ for all sectorial operators $A$, complex numbers $\alpha$ with $\operatorname{Re} \alpha>0$, and $t>0$.

3. Main results. In the following we assume the reader to be familiar with the basic theory of real interpolation spaces. Detailed information can be found in [4] or [16]. We will exclusively use the so-called "K-method". Since for certain parameters $\theta, p$ the spaces become trivial, we will profit 
from the following definition:

$$
(\theta, p) \in \Gamma: \Leftrightarrow\left\{\begin{array}{l}
\theta \in(0,1) \wedge 1 \leq p<\infty \text { or } \\
\theta \in[0,1] \wedge p=\infty
\end{array}\right.
$$

Let us state our first main result.

Theorem 1. Let $A \in \operatorname{Sect}(\omega), \varphi \in(\omega, \pi)$ and $\operatorname{Re} \alpha>0$. Take any function $0 \neq \psi \in \mathcal{O}\left(S_{\varphi}\right)$ such that $\psi, \psi z^{-\alpha} \in \mathcal{E}\left(S_{\varphi}\right)$. Then

$$
\left(X, \mathcal{D}\left(A^{\alpha}\right)\right)_{\theta, p}=\left\{x \in X \mid t^{-\theta \operatorname{Re} \alpha} \psi(t A) x \in \mathbf{L}_{*}^{p}((0, \infty) ; X)\right\}
$$

with the equivalence of norms

$$
\|x\|_{\left(X, \mathcal{D}\left(A^{\alpha}\right)\right)_{\theta, p}} \sim\|x\|_{X}+\left\|t^{-\theta \operatorname{Re} \alpha} \psi(t A) x\right\|_{\mathbf{L}_{*}^{p}((0, \infty) ; X)}
$$

for all $\theta \in(0,1), p \in[1, \infty]$.

The proof of Theorem 1 will be given in Section 6. Note that one could also allow the case $\theta=0, p=\infty$, for trivial reasons. However, it is important that the case $\theta=1, p=\infty$ is excluded. If one looks for conditions on $\psi$ which imply (4) also in this extremal case, one arrives at the following result.

Theorem 2. Let $A \in \operatorname{Sect}(\omega), \varphi \in(\omega, \pi)$ and $\operatorname{Re} \alpha>0$. Take a function $0 \neq \psi \in \mathcal{O}\left(S_{\varphi}\right)$ such that $\psi, \psi z^{-\alpha} \in \mathcal{E}\left(S_{\varphi}\right)$. Assume in addition that $\psi$ has the following properties:

(a) $\lim _{z \rightarrow 0} \psi(z) z^{-\alpha} \neq 0$

(b) $\psi(z) \neq 0$ for all $z \in S_{\varphi}$,

(c) $\sup _{z \in S_{\varphi}, s \geq 1}\left|\frac{\psi(s z)}{s^{\alpha} \psi(z)}\right|<\infty$.

Then

$$
\left(X, \mathcal{D}\left(A^{\alpha}\right)\right)_{\theta, p}=\left\{x \in X \mid t^{-\theta \operatorname{Re} \alpha} \psi(t A) x \in \mathbf{L}_{*}^{p}((0, \infty) ; X)\right\}
$$

with the equivalence of norms

$$
\|x\|_{\left(X, \mathcal{D}\left(A^{\alpha}\right)\right)_{\theta, p}} \sim\|x\|_{X}+\left\|t^{-\theta \operatorname{Re} \alpha} \psi(t A) x\right\|_{\mathbf{L}_{*}^{p}((0, \infty) ; X)}
$$

for all $(\theta, p) \in \Gamma$.

The proof of Theorem 2 will be given in Section 5 . The following special case is worth stating explicitly.

Theorem 3. Let $A \in \operatorname{Sect}(\omega), \varphi \in(\omega, \pi)$ and $\operatorname{Re} \alpha>0$. Take a function $0 \neq \psi \in \mathcal{O}\left(S_{\varphi}\right)$ such that $\psi, \psi z^{-\alpha} \in \mathcal{E}\left(S_{\varphi}\right)$. Assume in addition that $\psi$ satisfies the following conditions:

(a) $\lim _{z \rightarrow 0} \psi(z) z^{-\alpha} \neq 0$

(b) $\psi(z) \neq 0$ for all $z \in S_{\varphi}$,

(c) $\psi(\infty) \neq 0$. 
Then

$$
\left(X, \mathcal{D}\left(A^{\alpha}\right)\right)_{\theta, p}=\left\{x \in X \mid t^{-\theta \operatorname{Re} \alpha} \psi(t A) x \in \mathbf{L}_{*}^{p}((0, \infty) ; X)\right\}
$$

with the equivalence of norms

$$
\|x\|_{\left(X, \mathcal{D}\left(A^{\alpha}\right)\right)_{\theta, p}} \sim\|x\|_{X}+\left\|t^{-\theta \operatorname{Re} \alpha} \psi(t A) x\right\|_{\mathbf{L}_{*}^{p}((0, \infty) ; X)}
$$

for all $(\theta, p) \in \Gamma$.

Proof. One only has to show that also (c) of Theorem 2 is satisfied, at least on a slightly smaller sector. Choose $\varphi^{\prime} \in(\omega, \varphi)$. Then

$$
\left|\frac{\psi(s z)}{s^{\alpha} \psi(z)}\right|=\left|\frac{\psi(s z)(s z)^{-\alpha}}{\psi(z) z^{-\alpha}}\right| \leq\left\|\psi z^{-\alpha}\right\|_{\varphi}\left|\frac{1}{\psi(z) z^{-\alpha}}\right|
$$

and

$$
\left|\frac{\psi(s z)}{s^{\alpha} \psi(z)}\right| \leq\|\psi\|_{\varphi}\left|\frac{1}{\psi(z)}\right|
$$

independently of $s \geq 1$. The first right hand side above is bounded for $z \in S_{\varphi^{\prime}}$ with $|z| \leq 1$, the second for $z \in S_{\varphi^{\prime}}$ with $|z| \geq 1$.

In the next section we will give an independent proof of Theorem 3 . The main reason for doing so is that the proof in the special case is considerably easier and more elementary, and nevertheless shows some central ideas appearing also in the proof of the general result.

REMARK 3.1. The advantage of Theorems 2 and 3 is that they work also in the extremal case $\theta=1, p=\infty$. Moreover, one can already deduce the common descriptions (1)-(3) from them (see Sections 7.1 and 7.2.) However, they are unflexible in a certain sense. Namely, condition (a) of Theorem 2 prevents us from using the same function $\psi$ for describing interpolation spaces for different couples $\left(X, \mathcal{D}\left(A^{\alpha}\right)\right),\left(X, \mathcal{D}\left(A^{\beta}\right)\right)(\alpha \neq \beta)$. Theorem 1 is more flexible in this sense, and in fact implies reiteration results as, e.g.,

$$
\left(X, \mathcal{D}\left(A^{\alpha}\right)\right)_{\theta \frac{\operatorname{Re} \beta}{\operatorname{Re} \alpha}, p}=\left(X, \mathcal{D}\left(A^{\beta}\right)\right)_{\theta, p}
$$

where $0<\operatorname{Re} \beta \leq \operatorname{Re} \alpha, \theta \in(0,1), p \in[1, \infty]$ (cp. Corollary 7.3).

We conclude this section by noticing that one of the desired inclusions is easy.

Lemma 3.2. Let $A \in \operatorname{Sect}(\omega), \varphi \in(\omega, \pi)$ and $\operatorname{Re} \alpha>0$. Let $\psi, \widetilde{\psi}:=$ $\psi z^{-\alpha} \in \mathcal{E}\left(S_{\varphi}\right)$. Then

$$
\|\psi(t A) x\| \leq C K\left(t^{\operatorname{Re} \alpha}, x, X, \mathcal{D}\left(A^{\alpha}\right)\right) \quad(x \in X, t>0),
$$

where $C=\max \left(C_{\psi}, C_{\widetilde{\psi}}\right)$. Consequently, there is a continuous inclusion

$$
\left(X, \mathcal{D}\left(A^{\alpha}\right)\right)_{\theta, p} \subset\left\{x \in X \mid t^{-\theta \operatorname{Re} \alpha} \psi(t A) x \in \mathbf{L}_{*}^{p}((0, \infty) ; X)\right.
$$

for all $(\theta, p) \in \Gamma$. 
Proof. Let $x=a+b$ where $a \in X$ and $b \in \mathcal{D}\left(A^{\alpha}\right)$. Then $\psi(t A) x=$ $\psi(t A) a+t^{\alpha} \widetilde{\psi}(t A) A^{\alpha} b$. This yields

$$
\|\psi(t A) x\| \leq C_{\psi}\|a\|_{X}+t^{\operatorname{Re} \alpha} C_{\widetilde{\psi}}\left\|A^{\alpha} b\right\|_{X} \leq C\left(\|a\|_{X}+t^{\operatorname{Re} \alpha}\|b\|_{\mathcal{D}\left(A^{\alpha}\right)}\right) .
$$

Taking the infimum gives the first statement. The second follows readily.

4. Proof of Theorem 3. In this section we prove Theorem 3. The next result contains the key observation.

Proposition 4.1. Let $A \in \operatorname{Sect}(\omega), \varphi \in(\omega, \pi), \operatorname{Re} \alpha>0$ and $\psi, \psi z^{-\alpha} \in$ $\mathcal{E}\left(S_{\varphi}\right)$. Assume there are functions $f, g \in \mathcal{E}\left(S_{\varphi}\right)$ such that

$$
f \psi+g \psi z^{-\alpha}=1 \text {. }
$$

Then there is a constant $C \geq 1$ such that

$$
K\left(t^{\operatorname{Re} \alpha}, x, X, \mathcal{D}\left(A^{\alpha}\right)\right) \leq C\left(\|\psi(t A)\|+\min \left(t^{\operatorname{Re} \alpha}, 1\right)\|x\|\right)
$$

for all $x \in X$ and $t>0$.

Proof. Using (6) we define $h_{1}:=f \psi$ and $h_{2}:=g \psi z^{-\alpha}$. Then we write

$$
x=h_{1}(t A) x+h_{2}(t A) x=f(t A) \psi(t A) x+g(t A)\left(\psi z^{-\alpha}\right)(t A) x
$$

and observe that, by Lemma 2.3 , the vector $h_{2}(t A) x$ is in $\mathcal{D}\left(A^{\alpha}\right)$ with

$$
\left\|h_{2}(t A) x\right\|_{\mathcal{D}\left(A^{\alpha}\right)} \leq t^{-\operatorname{Re} \alpha} C_{g}\|\psi(t A) x\|+C_{h_{2}}\|x\| .
$$

Hence we obtain

$$
K\left(t^{\operatorname{Re} \alpha}, x, X, \mathcal{D}\left(A^{\alpha}\right)\right) \leq\left(C_{f}+C_{g}\right)\|\psi(t A) x\|+C_{h_{2}} t^{\operatorname{Re} \alpha}\|x\| .
$$

Now the statement follows from the inequality $K\left(t^{\operatorname{Re} \alpha}, x, X, \mathcal{D}\left(A^{\alpha}\right)\right) \leq\|x\|$, valid for all $x \in X$.

One should note that (6) already implies conditions (a)-(c) of Theorem 3. It is a surprising fact that these are already sufficient. To see this, we consider first a special case.

Lemma 4.2. Let $\operatorname{Re} \alpha>0$. Then there are functions $f, g \in \mathcal{O}\left(S_{\pi}\right)$ with

$$
f(z) \frac{z^{\alpha}}{(1+z)^{\alpha}}+g(z) \frac{1}{(1+z)^{\alpha}}=1 \quad\left(z \in S_{\pi}\right),
$$

and $f, g \in \mathcal{E}\left(S_{\varphi}\right)$ for each $0<\varphi<\pi$.

Proof. Choose $m \in \mathbb{N}$ with $m>2 \operatorname{Re} \alpha$. Multiply the equality $f z^{\alpha}+g=$ $(1+z)^{\alpha}$ by $(1+z)^{m-\alpha}$. This yields

$$
\begin{aligned}
f z^{\alpha}(1+z)^{m-\alpha}+g(1+z)^{m-\alpha} & =(1+z)^{m}=\sum_{j=0}^{m}\left(\begin{array}{c}
m \\
j
\end{array}\right) z^{j} \\
& =\sum_{j=0}^{k}\left(\begin{array}{c}
m \\
j
\end{array}\right) z^{j}+\sum_{j=k+1}^{m}\left(\begin{array}{c}
m \\
j
\end{array}\right) z^{j}
\end{aligned}
$$


where $k$ is chosen so that $\operatorname{Re} \alpha-1<k<m-\operatorname{Re} \alpha$, e.g., $k=\lfloor\operatorname{Re} \alpha\rfloor$. One can then define

$$
f(z):=\sum_{j=k+1}^{m}\left(\begin{array}{c}
m \\
j
\end{array}\right) \frac{z^{j-\alpha}}{(1+z)^{m-\alpha}}, \quad g(z):=\sum_{j=0}^{k}\left(\begin{array}{c}
m \\
j
\end{array}\right) \frac{z^{j}}{(1+z)^{m-\alpha}} .
$$

The following result, interesting in its own right, completes the proof of Theorem 3.

TheOREm 4. Let $\operatorname{Re} \alpha>0, \varphi \in(0, \pi)$ and $\psi \in \mathcal{O}\left(S_{\varphi}\right)$ satisfy

(a) $\psi, \psi z^{-\alpha} \in \mathcal{E}\left(S_{\varphi}\right)$,

(b) $\psi(z) \neq 0$ for all $z \in S_{\varphi}$,

(c) $\left.\psi(z) z^{-\alpha}\right|_{z=0} \neq 0$

(d) $\psi(\infty) \neq 0$.

Then there are functions $f, g \in \mathcal{O}\left(S_{\varphi}\right)$ such that

$$
f(z) \psi(z)+g(z) \psi(z) z^{-\alpha}=1 \quad\left(z \in S_{\varphi}\right),
$$

and $f, g \in \mathcal{E}\left(S_{\varphi^{\prime}}\right)$ for each $0<\varphi^{\prime}<\varphi$.

Proof. Applying Lemma 4.2 we find $f_{1}, g_{1}$ with $f_{1} z^{\alpha}+g_{1}=(1+z)^{\alpha}$. Then we write

$$
1=f_{1} \frac{z^{\alpha}}{(1+z)^{\alpha} \psi} \psi+g_{1} \frac{z^{\alpha}}{(1+z)^{\alpha} \psi} \psi z^{-\alpha}
$$

and it remains to show that $z^{\alpha} /(1+z)^{\alpha} \psi$ is in $\mathcal{E}\left(S_{\varphi}\right)$. But this is easy from the assumptions on $\psi$.

5. Proof of Theorem 2. It follows from Lemma 3.2 that we only have to care for the inclusion

$$
\left\{x \mid t^{-\theta \operatorname{Re} \alpha}\|\psi(t A) x\| \in \mathbf{L}_{*}^{p}(0, \infty)\right\} \subset\left(X, \mathcal{D}\left(A^{\alpha}\right)\right)_{\theta, p} .
$$

To establish it we want to write the constant $\mathbf{1}$ as a sum of appropriate functions $1=h_{1}+h_{2}$ as in Proposition 4.1. The weaker conditions on $\psi$, however, render this more complicated than in the previous section. We start with some auxiliary results.

Lemma 5.1. Let $\psi \in H_{0}^{\infty}\left(S_{\varphi}\right)$ and define

$$
h(z):=\int_{0}^{1} \psi(s z) \frac{d s}{s} \quad \text { and } \quad g(z):=\int_{1}^{\infty} \psi(s z) \frac{d s}{s} \quad\left(z \in S_{\varphi}\right) .
$$

Then $g, h \in \mathcal{E}\left(S_{\varphi}\right)$ with $h(0)=g(\infty)=0$. More precisely, if for some $\alpha>0$ the function $\psi z^{-\alpha}$ is bounded, so is $h z^{-\alpha}$, and if $\psi z^{\alpha}$ is bounded, so is $g z^{\alpha}$. 
If $x \in X$ is such that $\|\psi(t A) x\|_{X} \in \mathbf{L}_{*}^{1}(0,1)$ then

$$
h(A) x=\int_{0}^{1} \psi(s A) x \frac{d s}{s} .
$$

Proof. Since $\psi \in H_{0}^{\infty}$, the functions $g, h$ are well defined and bounded. By Morera's theorem, $g$ and $h$ are also holomorphic. Choose $C, \alpha>0$ such that $|\psi(z)| \leq C|z|^{\alpha}$. Then $|h(z)| \leq C|z|^{\alpha} \int_{0}^{1} s^{\alpha-1} d s$. This shows that $h$ is "good" at 0 . Analogously, $g$ is "good" at $\infty$. The function

$$
\gamma:=\int_{0}^{\infty} \psi(s z) \frac{d s}{s}
$$

is constant on $(0, \infty)$ (by change of variables), hence on the whole sector $S_{\varphi}$ (by holomorphy). Thus we can write $h-\gamma=g$, which shows $g, h \in \mathcal{E}\left(S_{\varphi}\right)$ and $g(0)=h(\infty)=\gamma$.

To prove the last assertion of the lemma, consider the approximants $h_{n}(z):=\int_{1 / n}^{1} \psi(s z) d s / s$. Standard arguments (namely, Fubini's theorem) and the assumption on $x$ show that

$$
h_{n}(A) x=\int_{1 / n}^{1} \psi(s A) x \frac{d s}{s} \rightarrow \int_{0}^{1} \psi(s A) x \frac{d s}{s} .
$$

On the other hand, with $C, \alpha>0$ as above, we have $\left|h_{n}(z)\right| \leq(C / \alpha)|z|^{\alpha}$ independently of $n$ and - for $m>\alpha$-Lebesgue's theorem yields

$$
(1+A)^{-m} h_{n}(A)=\left(\frac{h_{n}}{(1+z)^{m}}\right)(A) \rightarrow\left(\frac{h}{(1+z)^{m}}\right)(A)=(1+A)^{-m} h(A)
$$

in $\mathcal{L}(X)$. So we arrive at $(1+A)^{-m} \int_{0}^{1} \psi(s A) x d s / s=(1+A)^{-m} h(A) x$, which implies the desired statement.

REMARK 5.2. We note that the analogue of the last statement for $g$ is true if (and only if) $A$ is injective. We will not use this fact in what follows.

Lemma 5.3. Let $0 \neq \psi \in \mathcal{E}\left(S_{\varphi}\right)$ and $\operatorname{Re} \alpha>0$. Then there is $f \in$ $H_{0}^{\infty}\left(S_{\varphi}\right)$ such that

$$
\int_{0}^{\infty}(f \psi)(s z) \frac{d s}{s}=1 \quad\left(z \in S_{\varphi}\right)
$$

and $z^{\alpha} f \in H_{0}^{\infty}\left(S_{\varphi}\right)$.

Proof. Let $\bar{\psi}(z):=\overline{\psi(\bar{z})}$ and $\tau(z):=z /(1+z)^{2}$. Then $\psi(t) \bar{\psi}(t)=|\psi(t)|^{2}$ for all $t>0$. Choose $m>\operatorname{Re} \alpha$. Since $0 \neq \psi$,

$$
c:=\int_{0}^{\infty} \tau(s)^{m} \psi(s) \bar{\psi}(s) \frac{d s}{s}>0 .
$$

Then $f:=c^{-1} \tau^{m} \bar{\psi}$ is an appropriate choice. 
The next lemma states the well known Hardy-Young inequality.

Lemma 5.4 (Hardy-Young inequality). Let $\sigma>0, p \in[1, \infty]$ and $f$ : $(0, \infty) \rightarrow[0, \infty)$.

(a) If $t^{-\sigma} f \in \mathbf{L}_{*}^{p}(0, \infty)$ then $f \in \mathbf{L}_{*}^{1}(0, T)$ for every $T \in(0, \infty)$, and

$$
g(t):=\int_{0}^{t} f(s) \frac{d s}{s}
$$

satisfies $t^{-\sigma} g \in \mathbf{L}_{*}^{p}(0, \infty)$ and $\left\|t^{-\sigma} g\right\|_{\mathbf{L}_{*}^{p}(0, \infty)} \leq \sigma^{-1}\left\|t^{-\sigma} f\right\|_{\mathbf{L}_{*}^{p}(0, \infty)}$.

(b) If $t^{\sigma} f \in \mathbf{L}_{*}^{p}(0, \infty)$, then $f \in \mathbf{L}_{*}^{1}(T, \infty)$ for every $T \in(0, \infty)$, and

$$
g(t):=\int_{t}^{\infty} f(s) \frac{d s}{s}
$$

satisfies $t^{\sigma} g \in \mathbf{L}_{*}^{p}(0, \infty)$ and $\left\|t^{\sigma} g\right\|_{\mathbf{L}_{*}^{p}(0, \infty)} \leq \sigma^{-1}\left\|t^{\sigma} f\right\|_{\mathbf{L}_{*}^{p}(0, \infty)}$.

Proof. The second assertion follows from the first by a change of parameter $t \mapsto t^{-1}$. The first assertion can be proved easily by Riesz-Thorin interpolation since the case $p=\infty$ is trivial and the case $p=1$ is just the Fubini theorem. (Only positive operators are involved here, so interpolation is elementary, cf. [8].) Of course one can also look into a book, e.g., [11, pp. $245-246]$.

Let us turn to the key step.

Lemma 5.5. Let $A \in \operatorname{Sect}(\omega), \varphi \in(\omega, \pi), \operatorname{Re} \alpha>0$, and assume that $\psi \in \mathcal{E}\left(S_{\varphi}\right)$ satisfies all the hypotheses of Theorem 2 . Then there are functions $f \in H_{0}^{\infty}\left(S_{\varphi}\right)$ and $g \in \mathcal{E}\left(S_{\varphi}\right)$ such that

$$
\int_{0}^{1}(f \psi)(s z) \frac{d s}{s}+g(z) \psi(z) z^{-\alpha}=1 \quad\left(z \in S_{\varphi}\right) .
$$

Proof. We apply Lemma 5.3 to find a function $f \in H_{0}^{\infty}$ such that $\tilde{f}:=z^{\alpha} f \in H_{0}^{\infty}$ and $\int_{0}^{\infty}(f \psi)(s z) d s / s=1$ for all $z \in S_{\varphi}$. Let $h(z):=$ $\int_{1}^{\infty}(f \psi)(s z) d s / s$. From Lemma 5.1 it follows that $h \in \mathcal{E}$ and $h(0)=1$. Define $g(z):=h(z) / \psi(z) z^{-\alpha}$. Since by assumption $\left.\psi(z) z^{-\alpha}\right|_{z=0} \neq 0, g$ is "good" at 0 . To see that it is also "good" at $\infty$, we use condition (c) of Theorem 2 and write

$$
|g(z)|=\left|\frac{h(z)}{\psi(z) z^{-\alpha}}\right|=\left|\int_{1}^{\infty}(s z)^{\alpha} f(s z) \frac{\psi(s z)}{s^{\alpha} \psi(z)} \frac{d s}{s}\right| \leq C \int_{1}^{\infty}|\widetilde{f}(s z)| \frac{d s}{s},
$$

the latter being obviously "good" at $\infty$ (cf. Lemma 5.1).

The next result is parallel to Proposition 4.1 and is the last step in the proof of Theorem 2 . 
Proposition 5.6. Let $A \in \operatorname{Sect}(\omega), \varphi \in(\omega, \pi)$, $\operatorname{Re} \alpha>0$, and assume that $\psi, \psi z^{-\alpha} \in \mathcal{E}\left(S_{\varphi}\right)$. Assume furthermore that there are functions $f, g \in$ $\mathcal{E}\left(S_{\varphi}\right)$ with $(f \psi) \in H_{0}^{\infty}$ and

$$
1=\int_{0}^{1}(f \psi)(s z) \frac{d s}{s}+g(z) \psi(z) z^{-\alpha} \quad\left(z \in S_{\varphi}\right) .
$$

Then the conclusion of Theorem 2 holds.

Proof. As already noted, one inclusion is clear from Lemma 3.2. Let

$$
h_{1}(z):=\int_{0}^{1}(f \psi)(s z) \frac{d s}{s} \text { and } h_{2}(z):=g(z) \psi(z) z^{-\alpha} .
$$

Clearly, $h_{1}, h_{2} \in \mathcal{E}$. Since $z^{\alpha} h_{2}=\psi g \in \mathcal{E}$, Lemma 2.3 shows that $h_{2}(t A)$ maps $X$ into $\mathcal{D}\left((t A)^{\alpha}\right)=\mathcal{D}\left(A^{\alpha}\right)$ and

$$
A^{\alpha} h_{2}(t A) x=t^{-\alpha} g(t A) \psi(t A) x
$$

for all $x \in X$ and $t>0$. Hence we obtain

$$
\left\|h_{2}(t A) x\right\|_{\mathcal{D}\left(A^{\alpha}\right)} \leq t^{-\operatorname{Re} \alpha} C_{g}\|\psi(t A) x\|+C_{h_{2}}\|x\|
$$

for all $x \in X$ and $t>0$. This yields

$$
K\left(t^{\operatorname{Re} \alpha}, x, X, \mathcal{D}\left(A^{\alpha}\right)\right) \leq\left\|h_{1}(t A) x\right\|+C_{g}\|\psi(t A) x\|+t^{\operatorname{Re} \alpha} C_{h_{2}}\|x\| .
$$

Since also $K\left(t^{\operatorname{Re} \alpha}, x, X, \mathcal{D}\left(A^{\alpha}\right)\right) \leq\|x\|$, we can enlarge the constants to obtain

$$
K\left(t^{\operatorname{Re} \alpha}, x, X, \mathcal{D}\left(A^{\alpha}\right)\right) \leq C\left[\left\|h_{1}(t A) x\right\|+\|\psi(t A) x\|+\min \left(t^{\operatorname{Re} \alpha}, 1\right)\|x\|\right] .
$$

Now, take $(\theta, p) \in \Gamma, \theta \neq 0$ and $x \in X$ such that $t^{-\theta \operatorname{Re} \alpha}\|\psi(t A) x\| \in$ $\mathbf{L}_{*}^{p}(0, \infty)$. Then also $t^{-\theta \operatorname{Re} \alpha}\|(f \psi)(t A) x\| \in \mathbf{L}_{*}^{p}(0, \infty)$ since $\|(f \psi)(t A) x\| \leq$ $C_{f}\|\psi(t A) x\|$. By Hölder's inequality and $\theta \operatorname{Re} \alpha>0$ one has

$$
[s \mapsto(f \psi)(s t A) x] \in \mathbf{L}_{*}^{1}((0,1) ; X)
$$

for each $t>0$. Applying the second part of Lemma 5.1 yields

$$
\left\|h_{1}(t A) x\right\|=\left\|\int_{0}^{1}(f \psi)(s t A) x \frac{d s}{s}\right\| \leq \int_{0}^{t}\|(f \psi)(s A) x\| \frac{d s}{s}=: \sigma(t) .
$$

We can now apply the Hardy-Young inequality (Lemma 5.4) to obtain $t^{-\theta \operatorname{Re} \alpha} \sigma(t) \in \mathbf{L}_{*}^{p}(0, \infty)$ with

$$
\left\|t^{-\theta \operatorname{Re} \alpha} \sigma(t)\right\|_{\mathbf{L}_{*}^{p}} \leq \frac{1}{\theta \operatorname{Re} \alpha} C_{f}\left\|t^{-\theta \operatorname{Re} \alpha} \psi(t A) x\right\|_{\mathbf{L}_{*}^{p}(0, \infty)} .
$$

From (8) we can then infer $t^{-\theta \operatorname{Re} \alpha} K\left(t^{\operatorname{Re} \alpha}, x, X, \mathcal{D}\left(A^{\alpha}\right)\right) \in \mathbf{L}_{*}^{p}(0, \infty)$ and estimate the $\mathbf{L}_{*}^{p}$-norm of this function in terms of $\|x\|$ and $\left\|t^{-\theta \operatorname{Re} \alpha} \psi(t A) x\right\|_{\mathbf{L}_{*}^{p}}$.

Combining Lemma 5.5 and Proposition 5.6 completes the proof of Theorem 2 . 
6. Proof of Theorem 1. In this section we present the proof of Theorem 1. As before, the inclusion " $\subset$ " already follows from Lemma 3.2. In the proof of the other inclusion we will profit from the following auxiliary result.

Lemma 6.1. Let $A \in \operatorname{Sect}(\omega), \varphi \in(\omega, \pi), f \in H_{0}^{\infty}\left(S_{\varphi}\right)$ and $\operatorname{Re} \alpha>0$. Define

$$
h(z):=\int_{1}^{\infty} s^{-\alpha} f(s z) \frac{d s}{s} .
$$

Then $h \in H_{0}^{\infty}\left(S_{\varphi}\right)$ and

$$
h(A)=\int_{1}^{\infty} s^{-\alpha} f(s A) \frac{d s}{s} .
$$

Proof. If one chooses $0<\varepsilon<\operatorname{Re} \alpha$ small enough such that $z^{ \pm \varepsilon} f \in H^{\infty}$, one also has $z^{ \pm \varepsilon} h \in H^{\infty}$. This shows $h \in H_{0}^{\infty}$. The rest is simply Fubini's theorem and the definition of the $H_{0}^{\infty}$-functional calculus by the Cauchy integral. Note that the function $s \mapsto s^{-\alpha} f(s A)$ is in $\mathbf{L}_{*}^{1}((1, \infty) ; \mathcal{L}(X))$.

Returning to our main goal, we again try to write the constant $\mathbf{1}$ as a sum of appropriate functions. Hence we start as in the proof of Theorem 2 and choose a function $f \in H_{0}^{\infty}\left(S_{\varphi}\right)$ such that $\widetilde{f}:=z^{\alpha} f \in H_{0}^{\infty}\left(S_{\varphi}\right)$ and

$$
\int_{0}^{\infty}(f \psi)(s) \frac{d s}{s}=1
$$

(Lemma 5.3). Then we define

$$
h_{1}(z):=\int_{0}^{1}(f \psi)(s z) \frac{d s}{s} \text { and } h_{2}(z):=\int_{1}^{\infty}(f \psi)(s z) \frac{d s}{s} .
$$

By Lemma 5.1, $h_{1}, h_{2} \in \mathcal{E}\left(S_{\varphi}\right)$ and $h_{1}+h_{2}=1$. Moreover,

$$
z^{\alpha} h_{2}(z)=\int_{1}^{\infty} z^{\alpha} f(s z) \psi(s z) \frac{d s}{s}=\int_{1}^{\infty} s^{-\alpha}(\tilde{f} \psi)(s z) \frac{d s}{s}
$$

is - by Lemma 6.1 - a function in $H_{0}^{\infty}\left(S_{\varphi}\right)$. Then Lemma 2.3 shows that $h_{2}(t A)$ maps $X$ into $\mathcal{D}\left(A^{\alpha}\right)$ with

$$
\begin{aligned}
A^{\alpha} h_{2}(t A) & =t^{-\alpha}\left(z^{\alpha} h_{2}\right)(t A)=t^{-\alpha} \int_{1}^{\infty} s^{-\alpha}(\tilde{f} \psi)(s z) \frac{d s}{s}(t A) \\
& =\int_{1}^{\infty}(s t)^{-\alpha}(\tilde{f} \psi)(s t A) \frac{d s}{s}=\int_{t}^{\infty} s^{-\alpha}(\tilde{f} \psi)(s A) \frac{d s}{s}
\end{aligned}
$$

for all $t>0$ (use Lemma 6.1 again). 
Now, let $\theta \in(0,1), p \in[1, \infty]$ and take $x \in X$ such that $t^{-\theta \operatorname{Re} \alpha}\|\psi(t A) x\|$ $\in \mathbf{L}_{*}^{p}(0, \infty)$. By the above, $x=h_{1}(t A) x+h_{2}(t A) x$ and $h_{2}(t A) x \in \mathcal{D}\left(A^{\alpha}\right)$, hence

$$
K\left(t^{\operatorname{Re} \alpha}, x, X, \mathcal{D}\left(A^{\alpha}\right)\right) \leq\left\|h_{1}(t A) x\right\|+t^{\operatorname{Re} \alpha}\left\|h_{2}(t A) x\right\|+t^{\operatorname{Re} \alpha}\left\|A^{\alpha} h_{2}(t A) x\right\|
$$

for $t>0$. In the middle term we estimate $\left\|h_{2}(t A) x\right\| \leq C_{h_{2}}\|x\|$ andenlarging the constant-arrive at

$$
\begin{aligned}
& K\left(t^{\operatorname{Re} \alpha}, x, X, \mathcal{D}\left(A^{\alpha}\right)\right) \\
& \quad \leq C\left[\left\|h_{1}(t A) x\right\|+\min \left(t^{\operatorname{Re} \alpha}, 1\right)\|x\|+t^{\operatorname{Re} \alpha} \int_{t}^{\infty} s^{-\operatorname{Re} \alpha}\|\psi(s A) x\| \frac{d s}{s}\right] .
\end{aligned}
$$

Since we want to have $t^{-\theta \operatorname{Re} \alpha} K\left(t^{\operatorname{Re} \alpha}, x, X, \mathcal{D}\left(A^{\alpha}\right)\right) \in \mathbf{L}_{*}^{p}(0, \infty)$, the middle term is obviously good. The first term is dealt with exactly as in the proof of Proposition 5.6. For the third we define $g(s):=s^{-\operatorname{Re} \alpha}\|\psi(s A) x\|$ and observe that

$$
s^{(1-\theta) \operatorname{Re} \alpha} g(s)=s^{\theta \operatorname{Re} \alpha}\|\psi(s A) x\| \in \mathbf{L}_{*}^{p}(0, \infty)
$$

by assumption. Hence we can apply part (b) of the Hardy-Young inequality (Lemma 5.4), and we are done.

We note the following corollary to the proof.

Corollary 6.2. Let $A \in \operatorname{Sect}(\omega), \varphi \in(\omega, \pi), 0 \neq \psi \in \mathcal{E}\left(S_{\varphi}\right)$ and $\operatorname{Re} \alpha>0$. Then

$$
\int_{0}^{1} s^{-\operatorname{Re} \alpha}\|\psi(s A) x\| \frac{d s}{s}<\infty \Rightarrow x \in \mathcal{D}\left(A^{\alpha}\right) .
$$

Proof. Let $f, \tilde{f}, h_{1}, h_{2}$ be as before. Then $x=h_{1}(A) x+h_{2}(A) x$ and $h_{2}(A) x \in \mathcal{D}\left(A^{\alpha}\right)$. By assumption and Lemma 5.1, we have $h_{1}(A) x=$ $\int_{0}^{1} f(s A) \psi(s A) x d s / s$. As the operator $A^{\alpha}$ is closed and $A^{\alpha} f(s A) \psi(s A) x$ $=s^{-\alpha} \widetilde{f}(s A) \psi(s A) x$ is in $\mathbf{L}_{*}^{1}((0,1) ; X)$, one also has $h_{1}(A) x \in \mathcal{D}\left(A^{\alpha}\right)$ and

$$
A^{\alpha} x=\int_{0}^{\infty} \widetilde{f}(s A)\left[s^{-\alpha} \psi(s A) x\right] \frac{d s}{s} .
$$

7. Applications and comments. In this section we apply Theorem 2 to obtain the standard (and also new) descriptions of real interpolation spaces.

7.1. The Komatsu spaces. Let $A$ be a sectorial operator on the Banach space $X$ and let $\operatorname{Re} \alpha>0$. We consider the function

$$
\psi(z):=\frac{z^{\alpha}}{(1+z)^{\alpha}},
$$


which, by Lemma 2.1, satisfies the hypotheses of Theorem 3. An application of this theorem yields, after a change of variable $t \mapsto t^{-1}$, the description

$$
\left(X, \mathcal{D}\left(A^{\alpha}\right)\right)_{\theta, p}=\left\{x \in X \mid t^{\theta \operatorname{Re} \alpha}\left[A(t+A)^{-1}\right]^{\alpha} x \in \mathbf{L}_{*}^{p}(0, \infty)\right\}
$$

for all $(\theta, p) \in \Gamma$. If $0<\theta<1$, this characterization is connected to the name of Komatsu (see [12], [13] and compare also [17, Thm. 11.4.2]). The case $\alpha=1$ gives

$$
(X, \mathcal{D}(A))_{\theta, p}=\left\{x \in X \mid t^{\theta}\left[A(t+A)^{-1}\right] x \in \mathbf{L}_{*}^{p}(0, \infty)\right\},
$$

which is frequently used (see [15, Prop. 2.2.6]). The proofs of the cases $\alpha=1$ and $\alpha=2$ given in [16, Prop. 3.1.1 \& 3.1.6] were our motivating examples for Theorem 3 and served as a pattern for its proof.

7.2. Description by holomorphic semigroups. Let $A$ be a sectorial operator on the Banach space $X$ with $\omega_{A}<\pi / 2$. Choose $\varphi \in\left(\omega_{A}, \pi / 2\right)$. The function $f(z):=e^{-z}$ belongs to $\mathcal{E}\left(S_{\varphi}\right)$ and $f(t A)=e^{-t A}$ is the bounded holomorphic semigroup generated by $-A$. Let $\operatorname{Re} \alpha>0$. The function

$$
\psi(z):=z^{\alpha} e^{-z}
$$

has the property $\psi, z^{-\alpha} \psi \in \mathcal{E}\left(S_{\varphi}\right)$ and also satisfies (a) and (b) of Theorems 3 and 2. Condition (c) of Theorem 3 is not satisfied, but the more general condition (c) of Theorem 2 is:

$$
\frac{\psi(s z)}{s^{\alpha} \psi(z)}=\frac{e^{-s z}}{e^{-z}}=e^{-(s-1) z},
$$

and this is uniformly bounded for $z \in S_{\varphi}$ and $s \geq 1$. Hence Theorem 2 applies and yields the description

$$
\left(X, \mathcal{D}\left(A^{\alpha}\right)\right)_{\theta, p}=\left\{x \in X \mid t^{(1-\theta) \operatorname{Re} \alpha} A^{\alpha} e^{-t A} x \in \mathbf{L}_{*}^{p}(0, \infty)\right\}
$$

for all $(\theta, p) \in \Gamma$. This is also well known; see [16, Prop. 5.1.2] for the case $\alpha=m$ (which motivated our proof of Theorem 2).

7.3. Another characterization. Let again $A$ be a sectorial operator on the Banach space $X$ and consider the function

$$
\psi(z):=\frac{z^{\alpha}}{(1+z)^{\beta}}
$$

where $0<\operatorname{Re} \alpha<\operatorname{Re} \beta$. Then $\psi$ satisfies the conditions of Theorem 2 (but not those of Theorem 3 ). Hence, after changing variables, we obtain

$$
\left(X, \mathcal{D}\left(A^{\alpha}\right)\right)_{\theta, p}=\left\{x \in X \mid t^{\operatorname{Re} \beta-(1-\theta) \operatorname{Re} \alpha}\left[A^{\alpha}(t+A)^{-\beta}\right] x \in \mathbf{L}_{*}^{p}(0, \infty)\right\}
$$

for all $(\theta, p) \in \Gamma$. Specializing, e.g., $\alpha=1, \beta=2$ yields

$$
(X, \mathcal{D}(A))_{\theta, p}=\left\{x \in X \mid t^{1+\theta} A(t+A)^{-2} x \in \mathbf{L}_{*}^{p}(0, \infty)\right\} .
$$

This description may be new, at least we do not know of a reference. 
7.4. Interpolation inequalities and reiteration. We now demonstrate how our methods lead to proofs of important facts in the interpolation theory of sectorial operators. We start with the fact that the fractional domain space $\mathcal{D}\left(A^{\beta}\right)$ is of class $J(\theta) \cap K(\theta)$ between $X$ and $\mathcal{D}\left(A^{\alpha}\right)$, where $0<\operatorname{Re} \beta<\operatorname{Re} \alpha$ and $\theta:=\operatorname{Re} \beta / \operatorname{Re} \alpha$.

Corollary 7.1. Let $A$ be a sectorial operator and $0<\operatorname{Re} \alpha$. Then

$$
\left(X, \mathcal{D}\left(A^{\alpha}\right)\right)_{\theta, 1} \subset \mathcal{D}\left(A^{\beta}\right) \subset\left(X, \mathcal{D}\left(A^{\alpha}\right)\right)_{\theta, \infty}
$$

whenever $0<\operatorname{Re} \beta<\operatorname{Re} \alpha, \theta:=\operatorname{Re} \beta / \operatorname{Re} \alpha$.

Proof. Take $\varphi \in\left(\omega_{A}, \pi\right)$ and $0 \neq \psi \in \mathcal{O}\left(S_{\varphi}\right)$ with $\psi, z^{-\alpha} \psi \in \mathcal{E}\left(S_{\varphi}\right)$. The left hand inclusion follows from Theorem 1 and Corollary 6.2. To prove the right hand inclusion, observe $\widetilde{\psi}:=\psi z^{-\beta}$ is still in $\mathcal{E}$. Now, $\psi(t A) x=$ $t^{\beta} \widetilde{\psi}(t A) A^{\beta} x$, whence $\|\psi(t A) x\| \leq t^{\operatorname{Re} \beta} C_{\widetilde{\psi}}\left\|A^{\beta} x\right\|$ for all $t>0$. The rest is again Theorem 1.

It is well known that the left hand inclusion in (9) implies (and is in fact equivalent to) an inequality of the form $\left\|A^{\beta} x\right\| \leq C\|x\|^{1-\theta}\left\|A^{\alpha} x\right\|^{\theta}$, where $\theta:=\operatorname{Re} \beta / \operatorname{Re} \alpha$. The usual interpolation-theoretic proof of this implication requires a description of the interpolation spaces different from the K-method (cf. [4, Thm. 3.5.2] or [15, Thm. 1.2.15]). However, our methods allow also a direct proof.

Corollary 7.2 (Moment inequality). Let $A$ be a sectorial operator on the Banach space $X$. Let $\alpha, \beta, \gamma \in \mathbb{C}$ be such that $\operatorname{Re} \gamma<\operatorname{Re} \beta<\operatorname{Re} \alpha$ and $\operatorname{Re} \gamma>0$ or $\gamma=0$. Then there is a constant $C$ such that

$$
\left\|A^{\beta} x\right\| \leq \frac{C}{\theta(1-\theta)}\left\|A^{\gamma} x\right\|^{1-\theta}\left\|A^{\alpha} x\right\|^{\theta} \quad\left(x \in \mathcal{D}\left(A^{\alpha}\right)\right),
$$

where $\theta$ is defined as $\theta:=\frac{\operatorname{Re} \beta-\operatorname{Re} \gamma}{\operatorname{Re} \alpha-\operatorname{Re} \gamma}$.

Proof. Choose any $0 \neq \psi \in H_{0}^{\infty}$ such that $\psi z^{\alpha}, \psi z^{-\alpha}$ are still bounded functions. Define $h(z):=\int_{0}^{1} \psi(s z) d s / s, g(z):=\int_{1}^{\infty} \psi(s z) d s / s$ as in Lemma 5.1. Then $z^{-\alpha} h$ and $z^{\alpha} g$ are bounded functions, whence $\widehat{h}:=z^{-(\alpha-\beta)} h$ and $\widehat{g}:=z^{\beta-\gamma} g$ are both in $H_{0}^{\infty}$. For $x \in \mathcal{D}\left(A^{\alpha}\right)$ we have

$$
A^{\beta} x=h(t A) A^{\beta} x+g(t A) A^{\beta} x=t^{\alpha-\beta} \widehat{h}(t A) A^{\alpha} x+t^{-(\beta-\gamma)} \widehat{g}(t A) x .
$$

This yields

$$
\left\|A^{\beta} x\right\| \leq t^{\operatorname{Re} \alpha(1-\theta)} C_{\widehat{h}}\left\|A^{\alpha} x\right\|+t^{-\operatorname{Re} \alpha \theta} C_{\widehat{g}}\left\|A^{\gamma} x\right\|
$$

(see Lemma 2.2). Taking the infimum with respect to $t>0$ we arrive at

$$
\left\|A^{\beta} x\right\| \leq C\left[\left(\frac{1-\theta}{\theta}\right)^{\theta}+\left(\frac{\theta}{1-\theta}\right)^{1-\theta}\right]\left\|A^{\gamma} x\right\|^{1-\theta}\left\|A^{\alpha} x\right\|^{\theta},
$$

where $C:=\max \left\{C_{\widehat{g}}, C_{\widehat{h}}\right\}$. The term in brackets is bounded by $1 / \theta(1-\theta)$. 
The main part of the next result can be inferred from Corollary 7.1 by means of the so-called Reiteration Theorem from general interpolation theory (see [4, Thm. 3.5.3]). We present a different proof that uses essentially our Theorem 1.

COROLlary 7.3 (Reiteration). Let $A$ be a sectorial operator. Then the following assertions hold.

(a) If $0<\operatorname{Re} \beta \leq \operatorname{Re} \alpha$, then

$$
\left(X, \mathcal{D}\left(A^{\alpha}\right)\right)_{\theta \frac{\operatorname{Re} \beta}{\operatorname{Re} \alpha}, p}=\left(X, \mathcal{D}\left(A^{\beta}\right)\right)_{\theta, p}
$$

for all $\theta \in(0,1)$ and $p \in[1, \infty]$.

(b) If $0<\operatorname{Re} \gamma<\operatorname{Re} \beta \leq \operatorname{Re} \alpha, \sigma \in(0,1), p \in[1, \infty]$, and $x \in X$, then $x \in\left(X, \mathcal{D}\left(A^{\alpha}\right)\right)_{\theta, p} \Rightarrow x \in \mathcal{D}\left(A^{\gamma}\right)$ and $A^{\gamma} x \in\left(X, \mathcal{D}\left(A^{\beta-\gamma}\right)\right)_{\sigma, p}$,

where $\theta:=(1-\sigma) \frac{\operatorname{Re} \gamma}{\operatorname{Re} \alpha}+\sigma \frac{\operatorname{Re} \beta}{\operatorname{Re} \alpha}$.

(c) If $\alpha, \beta, \gamma, p, \sigma, \theta$ are as in (b), then

$$
\left(X, \mathcal{D}\left(A^{\alpha}\right)\right)_{\theta, p}=\left(\mathcal{D}\left(A^{\gamma}\right), \mathcal{D}\left(A^{\beta}\right)\right)_{\sigma, p} .
$$

Proof. To prove (a), simply choose a function $0 \neq \psi \in \mathcal{E}$ such that $z^{-\alpha} \psi \in H_{0}^{\infty}$. Then also $\psi z^{-\beta} \in H_{0}^{\infty}$ and one can apply Theorem 1 twice.

For the proof of $(\mathrm{b})$, choose $0 \neq \widetilde{\psi} \in \mathcal{E}$ such that $z^{-\alpha} \widetilde{\psi} \in \mathcal{E}$ and use it to describe $\left(X, \mathcal{D}\left(A^{\alpha}\right)\right)_{\theta, p}$. Observe that also $\psi:=z^{-\gamma} \widetilde{\psi} \in \mathcal{E}$ and $z^{-(\beta-\gamma)} \psi=$ $z^{-\beta} \widetilde{\psi} \in \mathcal{E}$. Hence we can use $\psi$ to describe $\left(X, \mathcal{D}\left(A^{\beta-\gamma}\right)\right)_{\sigma, p}$. (Note that $\theta, \sigma \in(0,1)$, whence Theorem 1 is applicable.) Now, since $\theta \operatorname{Re} \alpha-\operatorname{Re} \gamma>0$, we have

$$
\int_{0}^{1} s^{-\operatorname{Re} \gamma}\|\widetilde{\psi}(s A) x\| \frac{d s}{s}=\int_{0}^{1} s^{\theta \operatorname{Re} \alpha-\operatorname{Re} \gamma}\left\|s^{-\theta \operatorname{Re} \alpha} \widetilde{\psi}(s A) x\right\| \frac{d s}{s}<\infty
$$

if $x \in\left(X, \mathcal{D}\left(A^{\alpha}\right)\right)_{\theta, p}$. Applying Corollary 6.2 we see that $\left(X, \mathcal{D}\left(A^{\alpha}\right)\right)_{\theta, p} \subset$ $\mathcal{D}\left(A^{\gamma}\right)$. Moreover,

$$
t^{-\sigma \operatorname{Re}(\beta-\gamma)} \psi(t A) A^{\gamma} x=t^{-\theta \operatorname{Re} \alpha} \widetilde{\psi}(t A) x
$$

for all $t>0$. This finishes the proof of the stated equivalence.

Statement (c) follows immediately from (b) since here one can assume without restriction that $A$ is invertible.

These results are of course well known (see e.g. [17, Thm. 11.5.1] and compare this to statement (b) of Corollary 7.3). However, the method of proof is different.

7.5. A corona-type theorem. Last but not least, we would like to point out that Theorem 4 has the flavor of the "Corona Theorem", although it is not comparably deep. The general setting is algebraic in nature and quickly explained. One is given a domain $\Omega \subset \mathbb{C}$ and an algebra $\mathcal{A}$ of holomorphic 
functions on $\Omega$, containing 1 . For two given functions $f, g \in \mathcal{A}$ one wants to characterize (in function-theoretic terms) the following algebraic condition:

$$
\text { There exist } a, b \text { contained in } \mathcal{A} \text { such that } a f+b g=\mathbf{1} \text {, }
$$

i.e., the elements $f, g$ are relatively prime within the $\operatorname{ring} \mathcal{A}$. An obvious necessary condition is that $f, g$ do not have common zeroes. It is well known, but far from trivial, that if $\mathcal{A}=\mathcal{O}(\Omega)$ is the algebra of all holomorphic functions, this condition is also sufficient (see [19, Theorem 15.15]). If $\Omega=\mathbb{D}$ is the unit disc and $\mathcal{A}=H^{\infty}(\mathbb{D})$, then a necessary condition is $|f|+|g| \geq \delta$ for some $\delta>0$. That this condition is also sufficient is the result of Carleson and is the proper "Corona Theorem" (see [6]). Our Theorem 4 says that for $\mathcal{A}=\mathcal{E}\left(S_{\varphi}\right)$ and $a=\psi, b=z^{-\alpha} \psi$ the obvious necessary conditions for (10) are in fact sufficient.

Acknowledgements. This research was done while I enjoyed a oneyear stay at the Scuola Normale Superiore in Pisa as part of the EU-Research Training Network "Evolution Equations for Deterministic and Stochastic Systems" (HPRN-CT-2002-00281). I am indebted to Prof. Da Prato for giving me the opportunity to come to Pisa and for his constant support of my work. Also I would like to thank Prof. Ricci for pointing out to me the connection of Theorem 4 to the Corona Theorem.

\section{References}

[1] D. Albrecht, X. Duong, and A. McIntosh, Operator theory and harmonic analysis, in: Instructional Workshop on Analysis and Geometry, Part III (Canberra, 1995), Austral. Nat. Univ., Canberra, 1996, 77-136.

[2] W. Arendt, Semigroups and evolution equations: Functional calculus, regularity and kernel estimates, in: C. M. Dafermos and E. Feireisl (eds.), Handbook of Differential Equations, Elsevier, Amsterdam, 2004, 1-85.

[3] P. Auscher, A. McIntosh, and A. Nahmod, Holomorphic functional calculi of operators, quadratic estimates and interpolation, Indiana Univ. Math. J. 46 (1997), $375-403$.

[4] J. Bergh and J. Löfström, Interpolation Spaces. An Introduction, Grundlehren Math. Wiss. 223, Springer, Berlin, 1976.

[5] M. Cowling, I. Doust, A. McIntosh, and A. Yagi, Banach space operators with a bounded $H^{\infty}$-functional calculus, J. Austral. Math. Soc. Ser. A 60 (1996), 51-89.

[6] P. L. Duren, Theory of $H^{p}$ Spaces, Pure Appl. Math. 38, Academic Press, New York, 1970.

[7] K.-J. Engel and R. Nagel, One-Parameter Semigroups for Linear Evolution Equations, Grad. Texts in Math. 194, Springer, Berlin, 2000.

[8] M. Haase, Convexity inequalities for positive operators, submitted, 2004; preprint available at http://www.mathematik.uni-ulm.de/m5/aaapreprint/.

[9] —, A general framework for holomorphic functional calculi, Proc. Edinburgh Math. Soc. 48 (2005), 423-444. 
[10] M. Haase, The Functional Calculus for Sectorial Operators, book manuscript, to appear in: Oper. Theory Adv. Appl., Birkhäuser, Basel; preliminary version available at http://www.mathematik.uni-ulm.de/m5/haase, 2005.

[11] G. H. Hardy, J. E. Littlewood, and G. Pólya, Inequalities, Cambridge Math. Library, Cambridge Univ. Press, Cambridge, 1988. Reprint of the 1952 edition.

[12] H. Komatsu, Fractional powers of operators. II. Interpolation spaces, Pacific J. Math. 21 (1967), 89-111.

[13] -, Fractional powers of operators. VI. Interpolation of non-negative operators and imbedding theorems, J. Fac. Sci. Univ. Tokyo Sect. IA Math. 19 (1972), 1-63.

[14] P. C. Kunstmann and L. Weis, Maximal $L_{p}$-regularity for parabolic equations, Fourier multiplier theorems and $H^{\infty}$-functional calculus, in: Functional Analytic Methods for Evolution Equations (Levico Terme, 2001), Lecture Notes in Math. 1855, Springer, Berlin, 2004, 65-312.

[15] A. Lunardi, Analytic Semigroups and Optimal Regularity in Parabolic Problems, Prog. Nonlinear Differential Equations Appl. 16, Birkhäuser, Basel, 1995.

[16] -, Interpolation Theory, Appunti, Scuola Norm. Sup., Pisa, 1999.

[17] C. Martínez Carracedo and M. Sanz Alix, The Theory of Fractional Powers of Operators, North-Holland, Amsterdam, 2001.

[18] A. McIntosh, Operators which have an $H_{\infty}$ functional calculus, in: Miniconference on Operator Theory and Partial Differential Equations (North Ryde, 1986), Austral. Nat. Univ., Canberra, 1986, 210-231.

[19] W. Rudin, Real and Complex Analysis, 3rd ed., McGraw-Hill, New York, 1987.

Scuola Normale Superiore

Piazza dei Cavalieri 7

I-56126 Pisa, Italy

E-mail: haase@mathematik.uni-ulm.de

Received December 15, 2004

Revised version June 28, 2005 